\title{
Role of Fluid Injection in the Evolution of Fractured Reservoirs
}

\author{
James G. Berryman ${ }^{1, *}$ \\ ${ }^{1}$ University of California, Lawrence Berkeley National Laboratory, \\ One Cyclotron Road MS 74R316C, Berkeley, CA 94720, USA
}

(Dated: May 18, 2015)

\begin{abstract}
A survey is provided of some of the better known examples of quantitative results during fluid injection on number, quality, and weakening effects for fractures in earth reservoirs along with some comparisons to either well-known or better-known theories of both fracture arrival and/or new growth of existing fractures through both fluid injection and stress application. The detailed analyses presented focus on reservoirs having (at worst) orthotropic symmetry.
\end{abstract}

*JGBerryman@LBL.GOV 


\section{Introduction}

The following discussion is intended to highlight various aspects of fracture analysis as it may be applied to problems in the earth sceinces, and particularly in those cases where fluids (water, gas, oil, brine, $\mathrm{CO}_{2}$, etc.) may be involved. Certainly the importance of understanding how fluids effect the overall mechanical properties when they may be present in existing fractures in such systems is well-known, and of considerable practical importance. The additional role that fluids may play in affecting the overall behavior of these systems while being injected into existing fractures, or their role in either expanding or contracting such existing fractures, or creating new fractures is our main focus throughout.

\section{Some measures of scientific and/or engineering significance}

Many papers will be presented briefly in the following sections. It therefore seems both necessary and useful to comment briefly on how we might choose to grade or order the many papers discussed here. One popular method of doing so is to consider the number of times each paper has been cited in the literature by others. To account for this measure, the author has researched the citation histories of all the published papers being cited in this paper. However, it is still not clear how these data might be best presented. Should we show the raw numbers for the citations as of the writing of the present paper? Or should there be some normalization by the number of years since the publication of each paper? And how should very recent papers to treated, since they may have either no citations or very few citations as of the time of writing of the present work? The author has chosen NOT to normalize the paper citations by age, but rather to simply quote the numbers in cases where the papers have been in print long enough to accumulate some significant number of citations. In the case of the various newer papers, it was decided that all papers having at least 20 citations or more would have these numbers listed after the paper reference in the following bibliography. For papers with fewer than 20 citations as of the writing of the present paper, the resulting typically rather small number of citations is not being listed here. 


\section{Early Theories and Analyses of Fractured Systems}

Perhaps the earliest attempt to analyze the mechanics of fracture in solids was due to Griffith (1924). His work provides a wide-ranging discussion of fracture of various materials. His main example was glass rods, but he also discusses cracked plates, fibers, along with a brief treatment of applications to liquids. Sack (1946) (on page 730) corrects some mathematical errors in Griffith's paper, especially for materials containing circular cracks, and considers materials containing circular cracks. Sack's results may depend on Poisson's ratio of the material, whereas Griffith's results generally do not. Elliott (1947) generalized Griffith's approach for applications to metals, and provides a discussion of cracks in both 2D and 3D. This work concentrates especially on penny-shaped cracks and Griffith cracks. Results are in agreement with the experimental results of Griffith, but several differing versions of the formulas are also examined. Orowan (1949) presents an extensive review of the Griffith theory, a rederivation of those results based on atomic considerations, and also a detailed discussion about brittle strength of polycrystalline aggregates. However, the work of Brace (1960), which generalizes Griffith's approach and applies it to rocks, is one good example of work more pertinent to our present goals. Rice (1968) points out that the Griffith theory of elastic brittle fracture is also mathematically identical to the theory of fracture based on atomic cohesive forces as presented by Barenblatt (1962).

The works of Dugdale (1960) and Barenblatt (1962) are not themselves so directly pertinent to our targeted earth sciences problems, but they are nevertheless mentioned frequently in the references in later works by Rice (1968), Broberg (1971) and others, and therefore provide useful background for various related fracture analyses and applications.

Another early paper, and one that will play a major role in the later analyses of fluid effects here, is due to Athy (1930). This paper is the earliest one known to the author that introduces a nonlinear (or exponential) dependence into the formulas for mechanical behavior (i.e., elastic deformation) of soils. In particular, Athy shows that

$$
D=B+A\left(1-e^{-b x}\right)
$$

where $D$ is the density of the soil, $B$ is 1.4 - which is the density of a surface clay, $A$ is 1.3 - which is the maximum density of increase possible, $b$ is a constant, and $x$ is the depth of burial. 
Brace, W. F. (1960) provides useful information about early applications of Griffith's ideas (originally emphasizing applications to glass - but Griffith's discussion is more wide ranging than that) when applied to rocks. There is also a related preprint available online by Brace on the same topic, having the title: "Dependence of fracture strength of rocks on grain size."

Warren, J. E., and Root, P. J. (1963) is one of the classic references on double porosity modeling of reservoirs. Porosity is then assumed to be of two types: The first type is called primary porosity, being intergranular and controlled by deposition and lithification. It is highly interconnected and typically can be correlated with the permeability. The second type is called secondary porosity and is controlled by fracturing, jointing, or solution in circulating water. Secondary porosity is not highly interconnected, and therefore usually not correlated very well with the system permeability. They introduce the now well-known "sugar-cube model" with the matrix (being the primary regions containing porosity) composed of the cubes, and thin rough regions between these cubes being the locations of the fractures.

Broberg, K. B. (1971) gives an extensive discussion of stable and unstable crack growth in several areas of application, but mostly treats sheets of PVC, and metals. He quotes the Dugdale crack model extensively, and also the $J$ integral for crack applications due to Rice. His work compares and contrasts those results with the results of the Griffith energy approach to crack modeling.

Nilsson, F. (1973) emphasizes the fact that Rice's J-integral formulas are limited to static cases. So the work of Rice (1971), Broberg (1971), and others involving the J-integral cannot be applied directly in cases that involve dynamics, only statics and quasi-statics. However, he also shows how to modify these earlier analyses in order to include inertia effects in order to make the problems more realistic for a wider range of applications.

White, J. W. (1973) provides an efficient method of defining the failure surface in a numerical modeling code. He develops a systematic way of classifying and presenting material failure data for earth media that are approximately isotropic on the average. His functional is defined by

$$
I_{2 D}^{1 / 2}(f)=A_{1}\left(I_{1}\right)+A_{2}\left(I_{1}\right) I_{3 D}^{1 / 3}+A_{3}\left(I_{1}\right) I_{3 D}{ }^{2 / 3}
$$

where

$$
I_{1}=-\left(\sigma_{1}+\sigma_{2}+\sigma_{3}\right)
$$


which is three times the pressure.

$$
S_{i}=\sigma_{i}-\frac{1}{3}\left(\sigma_{1}+\sigma_{2}+\sigma_{3}\right)
$$

for $i=1,2,3$.

$$
\begin{gathered}
I_{2 D}^{1 / 2}=+\left\{(1 / 2)\left[\left(S_{1}\right)^{2}+\left(S_{2}\right)^{2}+\left(S_{3}\right)^{2}\right]\right\}^{1 / 2}, \\
I_{3 D}^{1 / 2}=\left(S_{1} \cdot S_{2} \cdot S_{3}\right)^{1 / 3} .
\end{gathered}
$$

The more recent work by Rubin (1991) is based in part on White's paper.

Another class of approximations that has repeatedly appeared in the earth sciences related literature is well-represented by the early paper of Budiansky and O'Connell (1976). This paper may well be the first to appear in the geophysics-related literature to reference the work of Bristow (1960) concerning the definition and make use of the "crack density parameters," which since then have become a recurring theme in many of the pertinent papers published on this topic. This paper introduced what are now standard ideas about how cracks influence the values of elastic constants and electrical conductivities in metals, and later works have applied the ideas to other materials including rocks. The work by Bristow has been used heavily in later works by Sayers and Kachanov (1991), Grechka and Kachanov (2006), Berryman and Grechka (2006), and many other followers of this line of work.

Tullis, J., and Yund, R. A. (1980) also discuss effects of temperature, strain, and strain rate on crack related problems.

Cheng, A. H.-D., and Liggett, J. A. (1984a) is the first of two papers by these authors on an efficient and accurate method for linear consolidation problems. They state their method is always preferred over other numerical methods - including finite element and finite difference methods — for such problems, whenever feasible.

Similarly, Cheng, A. H.-D., and Liggett, J. A. (1984b), present a second paper in their series, which uses the boundary integral equation method (based on a reciprocal principle) to formulate governing equations of Biot's linear porous-elasticity. Resulting fracture propagation criteria are then based on both elastic and plastic constitutive relations. Applications include failure of overconsolidated clay, earthquake prediction, and underground hydraulic fracturing for oil and gas exploration.

Tullis, J., and Yund, R. A. (1989) present their main result that trace amounts of water at high pressure can strongly enhance the rate of dislocation climb in deformed quartz 
aggregates. In general, the concentration of important water-related defects increases with pressure.

Rubin, M. R. (1991) presents a failure surface that is both simple (from the point of view of the algebra required) and isotropic. The paper gives examples of the use of the failure surface for both limestone and Monterey sand. The method is based in part on the earlier work of White (1973), which is quoted often. White's method is much simpler than (but also not as general as) Rubin's approach.

Sayers, C. M., and Kachanov, M. (1991) do not actually reference the work of Bristow (1960) in this paper, but it nevertheless depends heavily on ideas like those concerning crack density introduced earlier by Bristow (1960). These authors do give Bristow appropriate credit in their later works.

Johnson, P. A., and Rasolofosaon, P. N. J. (1996) actually has no equations, but includes many useful plots of laboratory data showing examples of nonlinear rock behavior.

Tuncay, K., and Corapcioglu, M. Y. (1996a) discuss some double-porosity models (such as those of Warren and Root) including the possibility of more than one fluid being present in the pores. The theory is strictly limited to linear pressure dependent behaviors however.

Tuncay, K., and Corapcioglu, M. Y. (1996b) continue the analysis of their previous paper by including some air-filled pores, and thus two-fluid modeling. Fast and slow compressional waves are included (as in Biot's theory), a second compressional wave arises due to the fractures, and a fourth compressional wave arises due to the pressure difference between the fluid phases in different porous blocks of their sugar-cube type model as also seen in Warren and Root (1963).

Tsvankin, I. (1997) does not treat nonlinear pressure effects, but does provide a uniform description of anisotropic media with either orthorhombic or transversely isotropic symmetry.

Wang, H. F. (1997) discusses various aspects of undrained compression in saturated sedimentary rock. In addition to Skempton's coefficients, the analysis also emphasizes Henkel's (1960) generalization of Skempton's coefficients, which is especially important for analysis of fully three-dimensional and polyaxial generalizations of Skempton's coefficients.

Vasco, D. W., and Karasaki, K. (2001) set up a numerical inversion scheme with equations that mostly involve linear integral equations. There can be some nonlinear interactions resulting from this formulation, but they are mostly covered within the complex formulation 
of the resulting integrals.

Berryman, J. G., Pride, S. R., and Wang, H. F. (2002) present a detailed analysis of a differential effective medium (DEM) scheme applied to rocks with either dry (gas-filled) or liquid-saturated fractures. Results show that as porosity tends to unity, the overall Poisson's ratio of these systems tends towards small positive values for the dry-fracture porous media, and thus tends towards one-half for the liquid-saturated cases. Examples treated include granites and sandstones.

Vasco, D. W. (2004) relates pressure and saturation changes to permeability variations within a reservoir. Theoretical estimates are shown to agree well with observed changes due to water and $\mathrm{CO}_{2}$ saturation changes.

Grechka, V., and Kachanov, M. (2006a,b) published two papers on rocks with closely spaced and intersecting cracks. The first paper performed a series of 3D finite element simulations on effective elasticity for systems that contain several sets of fractures embedded in otherwise isotropic host rock. Cracks might be close together, so that strong interactions among their stress fields may occur and the cracks may also overlap in some cases. They observed that the Noninteraction Approximation (NIA) nevertheless performed well in all the cases studied. In the second paper, they find that, while the theories of Schoenberg and Kachanov both fit finite-element computational results with comparable accuracy, the Kachanov approach appears to be the more useful one for fracture characterization. In particular, it correctly predicts the crack-induced anisotropy up to orthotropy. Other theories considered in the paper do not appear to predict this behavior quite as well.

Berryman, J. G., and Grechka, V. (2006) treats isotropic cracked systems as ensembles of cracked grains by making use of analogs to isotropic polycrystalline elastic media. They also make use of the numerical results from earlier work of Grechka and Kachanov (2006a,b) which included computer studies on sets of fractures embedded in otherwise isotropic host rock. Berryman and Grechka also found that the spread in values obtained was very comparable to those expected based on the Voigt (1928) and Reuss (1929) bounds. But the Hashin-Shtrikman(1961) bounds were generally found to be much too tight. On the other hand, the self-consistent (Berryman, 1992) estimates obtained at the same time were very good estimators of the average behavior of these systems.

Berryman, J. G., and Nakagawa, S. (2010) show how to deduce drained constants (for connected pores) from undrained (i.e., fluid-saturated pores, but the system of pores may not 
be very well interconnected) constants for general anisotropic systems having symmetries ranging from isotropic to orthotropic. Laboratory ultrasound data were inverted for the drained constants in three samples: one of glass beads, and two for angular sand grain packings. Experiments were performed under uniaxial stress, which produced hexagonal (i.e., transversely isotropic) symmetry for the poroelastic response. When the porosity, grain bulk modulus, and pore-fluid bulk modulus were known, the drained constants could then be uniquely determined.

Tsvankin, I., Gaiser, J., Grechka, V., van der Baan, M., and Thomsen, L. (2010) presents little detailed analysis here, but instead gives a general discussion of various issues involving data processing, and fracture characterization. This paper references other work mentioned here of Kachanov (1980) and Schoenberg and Sayers (1995).

Saevik, P. N., Berre, I., Jakobsen, M., and Lien, M. (2013) reconsider the asymmetric self-consistent, symmetric self-consistent, and differential schemes for permeability upscaling in fractured media. For open fractures, only the asymmetric method is seen to have correct asymptotic behavior. The asymmetric method is also found to predict percolation thresholds quite accurately.

Johnson, L. R. (2014) shows how seismic events are characterized in terms of first-degree moment tensors and interpreted in terms of a model combining shear cracks and wing cracks. Results can be used in either static or dynamic moment tensors.

As we shall see in the following discussion, such analyses have been broadly expanded upon in recent times by many researchers, especially when fluid injection plays an important role in the evolving fracture system behavior.

\section{Nonlinear Theories of Fluid-Enhanced Fracturing of Earth Materials}

After Athy (1930), the next well-known work on nonlinear response of earth materials via fluid injection is apparently that due to Wyble (1958). This author's main result is

$$
100 \frac{W_{p}}{W_{0}}-B=(100-B) 10^{-\alpha P}
$$

where $W_{0}$ is the value of the property of interest at zero pressure, $W_{P}$ is the value at pressure $P$, and $B$ is the per cent to which the curve is asymptotic. Symbol $\alpha$ is here the coefficient of the exponential decrease (determined from the slope of a semi-logarithmic plot). 
This result of Athy (1930) is similar to forms used later by Bandis, Lumsden, and Barton (1983) and also by Barton, Bandis, and Bakhtar (1985):

$$
V_{m}=A+B(J R C)+C\left(J C S / a_{1}\right)^{D}
$$

where the maximum fracture closure is $V_{m}, J R C$ is the joint closure strength, JCS is a measure of the joint roughness, $D$ is the exponent of the nonlinear term. Typical experimental values found through multiple regressions of data sets were $A \simeq-0.10$ to -0.30 , $B \simeq-0.007$ to $-0.006, C \simeq-1.01$ to -2.24 , and $D \simeq-0.23$ to -0.25 . Samples considered in the studies included slate, dolerite, limestone, siltstone, and sandstone, as well as samples from fresh, to moderately weathered, to weathered.

The result of Athy (1930) is also very similar to forms used later by Zimmerman et al. (1986), Schoenberg (2002), Daley et al. (2006), and Liu et al. (2009). For Zimmerman et al. (1986), the strain $\epsilon$ is given by:

$$
\epsilon=A P-B e^{-P / P^{*}}+D
$$

where $\mathrm{A}, \mathrm{B}, \mathrm{D}$, and $P^{*}$ are constants found by least-squares regression. For compressibility $C$, we have:

$$
C=A+\left(B / P^{*}\right) e^{-P / P^{*}}
$$

which is obtained by differentiating the previous equation with respect to pressure $P$. This result is commonly known and fairly widely used within the earth sciences community.

Han, D., Nur, A., and Morgan, D. (1986) introduces nonlinear behavior into the equations via

$$
V=B_{0}+B_{1} \phi+B_{2} \sqrt{c}+B_{3}\left[p-e^{-D p}\right]
$$

where $V$ is velocity, $p$ is pressure, $c$ is clay content, $\phi$ is porosity, and coefficients $B_{0}, B_{1}$, $B_{2}, B_{3}$, and $D$ are all curve-fitting parameters.

Zimmerman, R. W., Somerton, W., and King, M. S. (1986) find that the compressibility for three quarried, consolidated sandstones (Boise, Berea, and Bandera) is given generally by

$$
C=A+\left(B / P^{*}\right) e^{P / P^{*}}
$$

Constants $A$ and $B$ were found by least-squares regression. The parameter $P^{*}$ is found via a further nonlinear regression. 


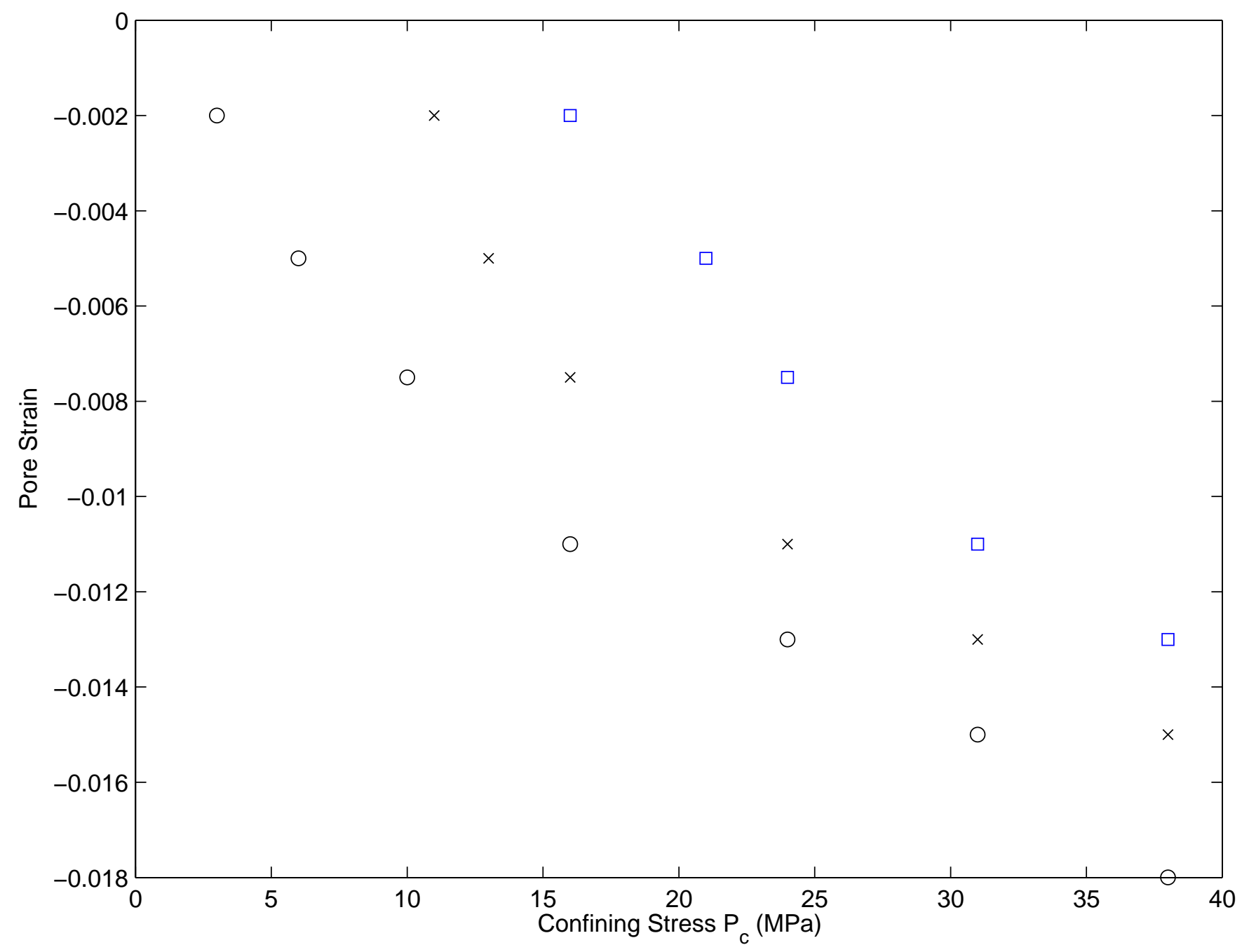

FIG. 1: Plot of pore strain versus confining stress using data from Zimmerman et al. (1986) for Boise Sandstone. Open circles are for $P_{p}=1.75 \mathrm{MPa}$, black x's are for $P_{p}=8.55 \mathrm{MPa}$, and black squares are for $P_{p}=15.45 \mathrm{MPa}$.

Figure 1 shows some plots of data on pressure-dependent fracture closure due to applied stress in Boise Sandstone as measured and discussed in this work by Zimmerman et al. (1986).

Brown, E. T., Bray, J. W., and Santarelli, J. T. (1989) also introduce nonlinear stressdependence into the formulas for the elastic constants related to boreholes and fractured earth systems. The pertinent equations for Young's modulus $E$ are

$$
E=\left(\alpha \sigma_{3}+b\right)^{m}
$$


and

$$
E=B-C e^{-A \sigma_{3}},
$$

where $B=E_{\infty}$ and $C=E_{\infty}-E_{0} . E_{0}$ and $E_{\infty}$ are respectively the values of $E$ when $\sigma_{3}=0$ and when $\sigma_{3} \rightarrow \infty$.

Eberhart-Phillips, D., Han, D.-H., and Zoback, M. D. (1989) established empirical relationships including two equations of the following form which were found for the compressional and shear wave velocities in sandstones:

$$
V=B_{0}+B_{1} \phi+B_{2} \sqrt{C}+B_{3}\left[P_{e}-e^{-D P e}\right],
$$

where $\phi$ is porosity, $C$ is clay content, and while $D$ and the $B$ 's are fitting constants, and $P_{e}$ is effective pressure.

Zimmerman, R. W. (1991) also treats the nonlinear case

$$
C_{b c}=C_{b c}^{\infty}+\left(C_{b c}^{i}-C_{b c}^{\infty}\right) e^{-P / P^{\infty}},
$$

where $C_{b c}$ is the zero pressure compressibility, $C_{b c}^{\infty}$ is the asymptotic high pressure compressibility when cracks are closed. $P^{\infty}$ is a pressure scaling factor determining the limiting value of the compressibility. This equation is based in part on Wyble (1958) and in part on Zimmerman et al. (1986).

Matsuki, K., et al (2001), study the nonlinear effects of crack closure in granite under normal stress conditions. They show that the elastic closure $\Delta$ is approximately linear in the logarithm of normal stress $\sigma_{n}$. So we have

$$
\Delta=A+B \ln \sigma_{n}
$$

with $A$ and $B$ being fitting constants. Time dependent closure is then given by

$$
\Delta(\infty)-\Delta(0)=B \ln \frac{Y_{E}(0)}{Y_{E}(\infty)} .
$$

Figure 2 shows some plots of data on time-dependent fracture closure due to applied stress in Inada Granite as discussed in the work by Matsuki et al. (2001).

From Schoenberg (2002), we have:

$$
Z_{N}(\theta)=Z_{N_{\infty}}(\theta)+\left[Z_{N_{0}}-Z_{N_{\infty}}(\theta)\right] e^{-\tau_{\perp}(\theta) / \tau_{N}(\theta)}
$$




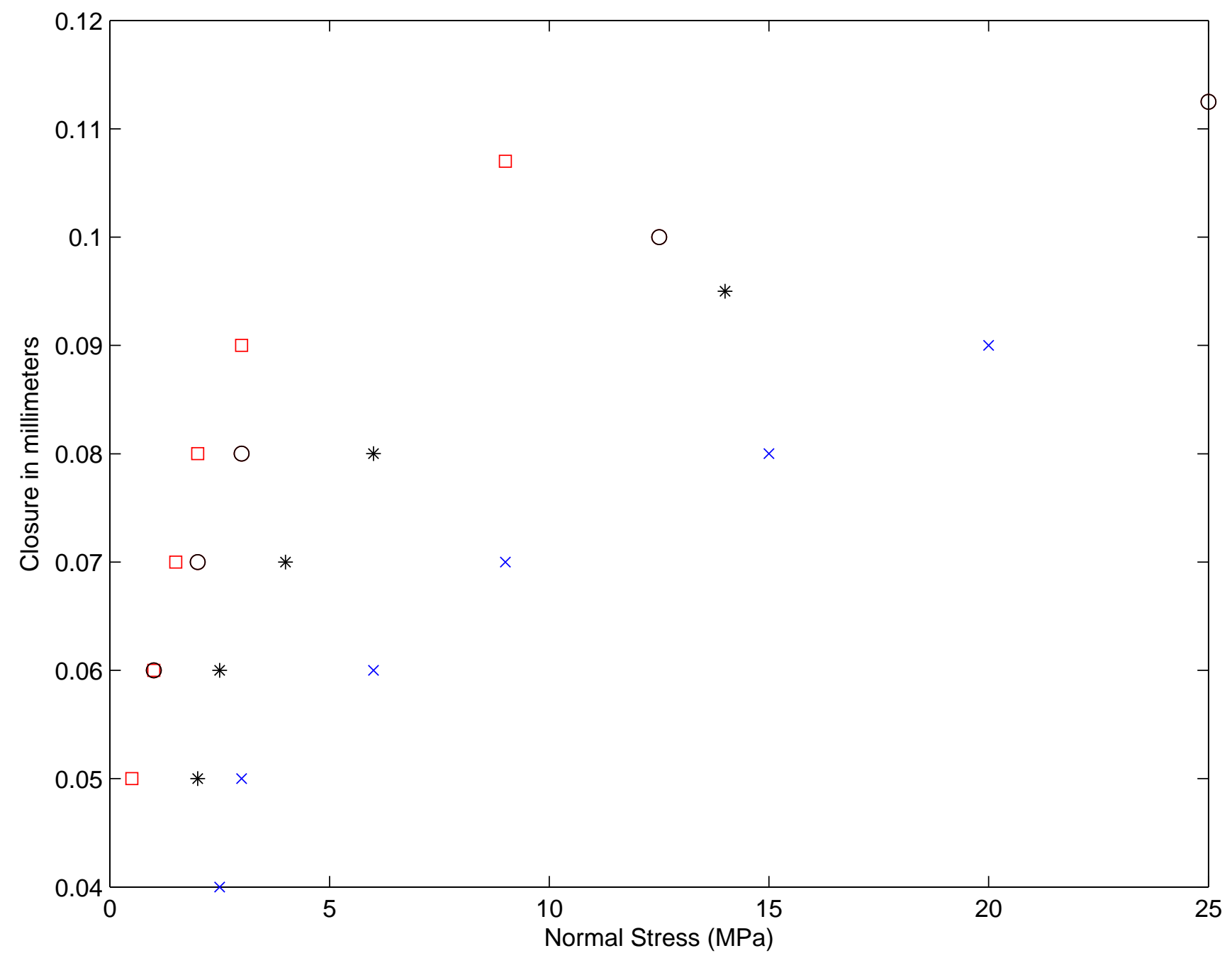

FIG. 2: Plot of crack closure versus applied stress for data from Matsuki et al. (2001) for Inada Granite (IG). Red squares are for sample 10, black *'s are for sample 15, blue x's are for sample 20 , and green triangles are for sample 25 .

and

$$
Z_{T}(\theta)=Z_{T_{\infty}}(\theta)+\left[Z_{T_{0}}-Z_{T_{\infty}}(\theta)\right] e^{-\tau_{\perp}(\theta) / \tau_{T}(\theta)} .
$$

where $Z_{N}$ and $Z_{T}$ are constants for excess normal stress and excess tangential stress, respectively.

Malama, B., and Kulatilake, P. H. S. M. (2003) show that the rate of decrease of the jointed rock normal compliance, $C_{j}$, with increasing normal compressive stress $\sigma$, is expressed in formulas as: $\partial C_{j} / \partial \sigma=-f(\sigma)$, where $f(\sigma)=a e^{-b \sigma}$ is a function determined in the presented analysis. The relation between the total deformation $v$ and the normal stress $\sigma$ is 


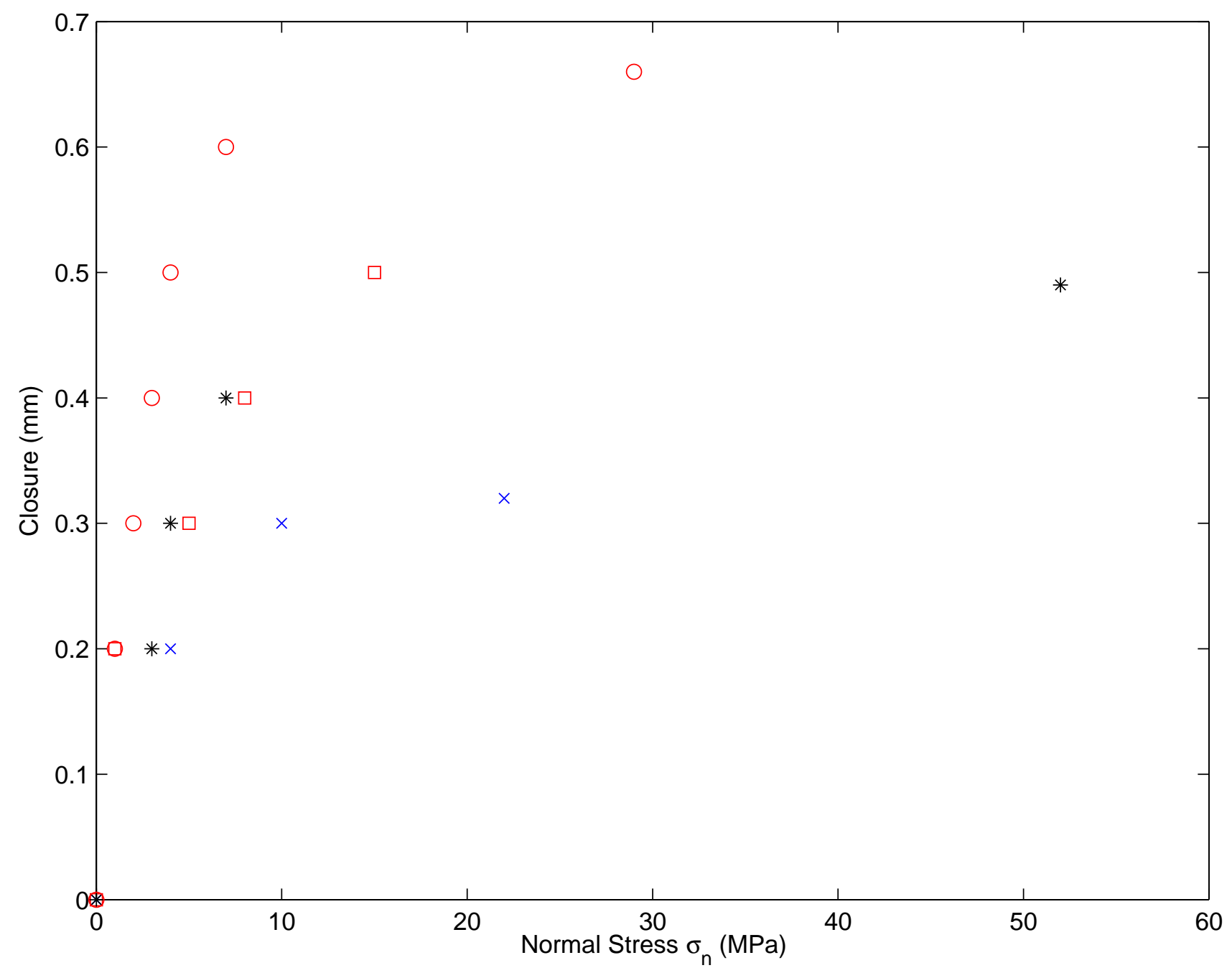

FIG. 3: Plot of crack closure versus applied stress for data from Malama and Kulatilake (2003) for Diorite and Granodiorite rock. Blue x's are for sample Dr-1, red squares are for Grd-1, blue *'s are for Grd-2, and black circles are for Grd-3.

given by

$$
v=\frac{a}{b^{2}}\left[1-e^{-b \sigma}\right]+C_{i} \sigma,
$$

where $C_{i}$ is the limiting value as $\sigma \rightarrow \infty$. And

$$
\Delta v=\Delta v_{m}\left[1-e^{\left.-\left(\sigma / \sigma_{1 / 2}\right) \ln 2\right)}\right]
$$

where $\Delta v_{m}$ is the maximum joint closure in the limit as $\sigma \rightarrow \infty$, and given by $\Delta v_{m}=a / b^{2}$, and $b=(\ln 2) / \sigma_{1 / 2}$, where $\Delta v_{m} / 2$ determines the value of $\sigma_{1 / 2}=\sigma\left(\Delta v_{m} / 2\right)$.

Figure 3 shows the types of behavior observed by Malama and Kultatilake (2003). 
Neuzil, C. E. (2003), makes good use of the earlier work of Athy (1930). See (1).

Shapiro, S. A. (2003) shows that, for compressibility, we have

$$
C_{d r}(P)=C_{d r s}\left[1-\theta_{s}\left(C_{d r s}-C_{g r}\right) P+\theta_{c} \phi_{c 0} \exp \left(-\theta_{c} P C_{d r s}\right)\right]
$$

And for bulk modulus:

$$
K_{d r}(P)=K_{d r s}\left[1+\theta_{s}\left(C_{d r s}-C_{g r}\right) P-\theta_{c} \phi_{c 0} \exp \left(-\theta_{c} P C_{d r s}\right)\right]
$$

Here $K_{d r s}$ and $C_{d r s}$ are related by

$$
K_{d r s}=1 / C_{d r s}
$$

The skeleton shear modulus is given by

$$
\mu_{d r}(P)=\mu_{d r s}\left[1+\theta_{s \mu}\left(C_{d r s}-C_{g r}\right) P-\theta_{c \mu} \phi_{c 0} \exp \left(-\theta_{c} P C_{d r s}\right)\right]
$$

The shear modulus of the dry rock is $\mu_{d r}$ in the case of closed compliant porosity, while the stiff porosity is equal to $\phi_{s 0}$, and

$$
\theta_{s \mu}=-\frac{1}{\mu_{d r s}} \frac{\partial \mu_{d r}}{\partial \phi_{s}}, \quad \theta_{c \mu}=-\frac{1}{\mu_{d r s}} \frac{\partial \mu_{d r}}{\partial \phi_{c}} .
$$

The quantity $\theta_{s}$ can also be estimated using the Hashin-Shtrikman upper bound for $\mu_{d r}$, which is given approximately by:

$$
\theta_{s \mu} \simeq 1+\frac{6 K_{g r}+2 \mu_{g r}}{9 K_{g r}+8 \mu_{g r}} .
$$

Similarly, Daley et al. (2006) show that:

$$
\begin{gathered}
Z_{T}=Z_{T_{\infty}}+\left[Z_{T_{0}}-Z_{T_{\infty}}\right] e^{-\sigma_{11} / \tau_{T}}, \\
Z_{N}=Z_{N_{\infty}}+\left[Z_{N_{0}}-Z_{N_{\infty}}\right] e^{-\sigma_{11} / \tau_{N}},
\end{gathered}
$$

where $Z_{N}$ and $Z_{T}$ again are constants for excess normal stress and excess tangential stress, respectively. Then, the fracture permeability $k_{T}$ is also determined by

$$
k_{T}=k_{T_{\infty}}+\left[k_{T_{0}}-k_{T_{\infty}}\right] e^{-\sigma_{11} / \tau_{k}}
$$

The decay constants for $Z_{T}, Z_{N}$, and $k_{T}$ are $\tau_{T}, \tau_{N}$, and $\tau_{k}$, respectively. This paper also references earlier work by Bandis, Lumsden, and Barton (1983), and by Barton, Bandis, and Bakhtar (1985). 
Chen, C. Y., and Horne, R. N. (2006) treat rough-walled fractures and two-phase (specifically, water and gas) flow problems. They found that their tortuous channel model (or TCM) could be expressed via relative permeabilities given by

$$
k_{r w}=0.74 S_{w}^{* 2}+0.26 S_{w}^{*}
$$

and

$$
k_{r g}=0.43 S_{g}^{3}+0.38 S_{g}^{2}+0.19 S_{g}
$$

where subscripts $w$ and $g$ stand for water and gas respectively, with $S_{w}$ and $S_{g}$ being their respective saturations.

Einav, I. (2007a) introduces the concept of "breakage" in the context of granular materials. This work distinguishes between the older "damage mechanics" that had been developed by others for describing decreases in stiffness and strength of "solid-like" materials (unlike granular materials that are assumed to have many distinct grains and gaps between and among the grains' surfaces when we begin our analysis). Relative breakage $B_{r}$ is defined by

$$
B_{r}=B_{t} / B_{p}
$$

where $B_{p}$ is breakage potential, and $B_{t}$ is total breakage. Thus, $0 \leq B_{r} \leq 1$. The paper gives detailed comparisons to earlier work on both damage mechanics and breakage analysis. He calls this approach "continuum breakage mechanics" or CBM.

Einav, I. (2007b) continues the work of the previous paper by discussing both pure elasticbreakage theory and elastic-plastic breakage. There are examples of comparisons between the proposed theory and experiments on well-graded sands, among several others. Expressions for elastic compressional free energy $e_{v}^{e}$ are given by

$$
e_{v}^{e}-e_{v 0}=\frac{\left(p / p_{r}\right)^{1-m}-1}{K(1-m)},
$$

where $e_{v 0}$ is an initial volumetric strain, while $m$ and $K$ are material constants. $m$ typically ranges from $1 / 3$ to $1 / 2$, while taking $m \rightarrow 1$ results in a logarithmic form of this equation.

Zhang, Y., Sayers, C. M., and Adachi, J. I. (2009) derived the following equations which were based in part on the work of Schoenberg (2002):

$$
B_{N}=B_{N_{\infty}}+\left[B_{N_{0}}-B_{N_{\infty}}\right] e^{-\sigma_{n e f f} / \tau_{N}}
$$

and

$$
B_{T}=B_{T_{\infty}}+\left[B_{T_{0}}-B_{T_{\infty}}\right] e^{-\sigma_{n e f f} / \tau_{T}}
$$


where $B_{N}$ and $B_{T}$ are the normal and shear compliances of the fractures. $\sigma_{n e f f}$ is the normal effective stress acting normal to the fracture. $\tau_{N}$ and $\tau_{T}$ are characteristic stresses that determine the rate of $B_{N}$ and $B_{T}$ with increasing effective stress $\sigma_{n e f f}$.

Salimi, H., and Bruining, H. (2010), continue the analysis of Warren and Root (1963) to include some air-filled pores, and thus two-fluid modeling. Fast and slow compressional waves are included (as in Biot's theory), a second compressional wave arises due to the fractures, and a fourth compressional wave arises due to the pressure difference between the fluid phases in different porous blocks of their sugar-cube type model.

From Liu et al. (2009), we have bulk rock compressibility $C_{b c}=\frac{1}{K}$ determined by:

$$
C_{b c}=C_{b c}^{\infty}+\left(C_{b c}^{i}-C_{b c}^{\infty}\right) \exp (-\sigma / P)
$$

where $P$ is some characteristic (or reference) pressure or stress, $i$ indicates the initial value of the bulk rock compressiblity $C_{b c}$ which is then defined by (38), where $\sigma$ is the applied stress. This work was based in part on earlier work by Wyble (1958), as well as that of Zimmerman et al. (1986), Zimmerman (1991), and a few others as quoted in the paper.

This work was then later extended by Liu and Rutqvist (2013), who generalized the work of Liu et al. (2009) to multiphase flow in a dual-continuum system, where the second phase (t) contains cracks or fractures, and the first phase (e) involves harder (perhaps rounder and therefore stiffer) pores. Liu, Wei, and Rutqvist (2013) also show that, if $b$ is the averaged fracture aperture, then

$$
b=b_{0, e}\left(1-\frac{\sigma_{n}}{K_{F, e}}\right)+b_{0, t} \exp \left(-\frac{\sigma_{n}}{K_{F, t}}\right),
$$

where label $F$ is fracture, label 0 is for the unstressed state, label $e$ is for the hard part, and label $t$ is for the soft part. They also found that computed results using these formulas agree well with several previously published data sets.

Das, A., Nguyen, G. D., and Einav, I. (2011) show that the Helmholtz free energy is:

$$
H=(1-\theta B)(1 / 2)\left[K\left(\epsilon_{v}^{e}\right)^{2}+3 G\left(\epsilon_{s}^{e}\right)^{2}\right]
$$

where $B$ is the internal variable for continuum breakage, $K$ is bulk modulus, $G$ is shear modulus, and

$$
p=(1-\theta B) K \epsilon_{v}^{e}, \quad q=3(1-\theta B) G \epsilon_{s}^{e} .
$$

Here $p$ is mean effective stress, $q$ is shear stress, $\epsilon_{v}$ is volumetric strain, elastic volumetric strain is $\epsilon_{v}^{e}$, and elastic shear strain is $\epsilon_{s}^{e}$. For shear related dissipation, they adopt either 
the Hoek-Brown failure criterion, or the Mohr-Coulomb failure criterion. Then, breakage energy is given by

$$
E_{B}=\left[\theta / 2(1-\theta B)^{2}\right]\left(p^{2} / K+q^{2} / 3 G\right)
$$

Rubin, M. B., and Einav, I. (2011) show that the effective bulk and shear moduli are given by

$$
K_{e f f} \simeq k(1-\phi)^{3 / 2}\left[(1-\theta B) p_{1} / k\right]^{1 / 2}
$$

and

$$
G_{e f f} \simeq(g / k) K_{e f f}
$$

where $\phi$ is porosity, $B$ is the breakage coefficient, $\theta$ is a grading index, and $p_{1}$ is pressure. The scaling constants for shear a bulk modulus are $g$ and $k$.

Lian, P. Q., Cheng, L. S., and Liu, L. F. (2011) finds that

$$
k=k_{0} e^{-\alpha_{0}\left(p_{0}-p\right)}
$$

where $k_{0}$ is initial permeability, $k$ is permeability under stress, $p_{0}$ is initial pressure, $\alpha_{0}$ is the deformation coefficient, and $p$ is applied pressure. This analysis treats the region outside the wellbore as having a closed zone near the wellbore, and a fractured zone outside the closed zone.

David, E. C., and Zimmerman, R. W. (2012) discuss the gradual closure of compliant pores under stress, and the remaining influence of stiff non-closable pores at high-pressure. They also incorporate results from the differential scheme for the resulting changes in values for the elastic moduli.

Sayers, C. M., and den Boer, L. D. (2012) show that normal and shear compliances are given by

$$
B_{N}\left(\Delta \sigma_{n}^{\prime}\right)=B_{N}^{0} e^{-\Delta \sigma_{n}^{\prime} / \sigma_{c}}
$$

and

$$
B_{T}\left(\Delta \sigma_{n}^{\prime}\right)=B_{T}^{0} e^{-\Delta \sigma_{n}^{\prime} / \sigma_{c}}
$$

The characteristic stress determining the rate of decrease of fracture compliance with increasing normal compressive stress is given by $\sigma_{c}$. These equations were also derived in part based on the work of Schoenberg (2002). 
Bunger, A. P., Gordeliy, E., and Detournay, E. (2013) present data and simulations on hydraulic fractures that tend to grow at shallow depths towards the surface, or in more general situations they curve towards any free surface that happens to be near the fracture.

Katsuki, D., Gutierrez, M., and Almrabat, A. (2014) incorporates into its main equations the same ones as those of presented earlier by Zimmerman et al. (1986), and Han et al. (1986).

\section{Fluid Injection into Orthotropic Fractured Reservoirs}

Berryman (2012) treats the poroelastic behavior of orthotropic fractured reservoirs. Our focus here is on fluid injection and how such fluid injection might lead to new or growing fractures. But normal fluids cannot support the storage of any shear energy. Recall [see Mavko et al., (1998)] that an orthorhombic system under hydrostatic stress, or under uniaxial stress applied parallel to the axis of symmetry, or under triaxial stress applied parallel to be axis of symmetry will in all these cases still result in a system also having orthorhombic symmetry. (There remain a few cases in which the resulting symmetry could be either monoclinic or triclinic, but we shall exclude these from consideration here.) So the resulting poroelastic equations we need to use for such applications to orthotropy can all be simplified to:

$$
\left(\begin{array}{c}
e_{11} \\
e_{22} \\
e_{33} \\
-\zeta
\end{array}\right)=\left(\begin{array}{cccc}
S_{11} & S_{12} & S_{13} & -\beta_{1} \\
S_{12} & S_{22} & S_{23} & -\beta_{2} \\
S_{13} & S_{23} & S_{33} & -\beta_{3} \\
-\beta_{1} & -\beta_{2} & -\beta_{3} & \gamma
\end{array}\right)\left(\begin{array}{c}
\sigma_{11} \\
\sigma_{22} \\
\sigma_{33} \\
-p_{f}
\end{array}\right),
$$

where the $e_{i i}$ (no summation over repeated indices) are strains in the $i=1,2,3$ directions. The $\sigma_{i i}$ are the corresponding stresses. The fluid pressure is $p_{f}$. The increment of fluid content is $\zeta$, which (like the strains) is dimensionless. The drained compliances are $S_{i j}^{d} \equiv S_{i j}$. The drained Reuss average bulk modulus is then defined via

$$
\frac{1}{K_{R}^{d}} \equiv \sum_{i j=1,2,3} S_{i j}^{d},
$$

a quantity which is the one commonly taken to be the definition of the bulk modulus of such a simple (non-heterogeneous) anisotropic system. Undrained compliances will be symbolized by $S_{i j}^{u}$. For the Reuss average undrained bulk modulus $K_{R}^{u}$, we have drained compliances 
replaced by undrained compliances in a formula otherwise identical in form to (49). Offdiagonal coefficients $\beta_{i}=S_{i 1}+S_{i 2}+S_{i 3}-1 / 3 K_{R}^{g}$, where $K_{R}^{g}$ is again the Reuss average modulus of the grains - simply replace $d$ 's with $g$ 's in (49) to determine $K_{R}^{g}$.

One good choice of the resulting changes in the fracture aperature is then given by (39). Both the hard and soft porosities are then taken into account along with their appropriate weighting factors, as well as sensitivities to the known distinct sources of pressure effects.

\section{Fluid Injection and Fracture Extension}

Zhou, X., and Burbey, T. J. (2014) provide a recent review and extensive survey of ways in which fluid injection may influence both fracture initiation and fracture propagation. These authors provide a listing and discussion of 78 pertinent references. We will emphasize a smaller subset of these works here, including only those that are most pertinent to our present goals.

Howard, G. C., and Fast, C. R. (1957) discuss optimal fluid characteristics for successful fracture extension. They emphasize a coefficient $C$ called the fracturing-fluid coefficient. This coefficient is defined in relationship to three types of linear flow mechanisms, which are: (a) viscosity and relative-permeability effects, (b) reservoir-fluid viscosity-compressibility effects, and (c) wall-building effects. They show results for the effect of fracturing-fluid viscosity on area of fracture, for the effect of reservoir fluid on area of fracture, and for the effect of fracturing-fluid coefficient and pump rate on fracture extent, among a total of eight figures.

Rice, J. R., and Cleary, M. P. (1976) study fluid-saturated porous media with compressible constituents while emphasizing stress-induced diffusion effects. This work is so well-known and also sufficiently complicated that we will not attempt to summarize their main results here.

Zoback, M. D., et al. (1977) present results of hydraulic fracturing experiments on both intact and prefractured rocks in a laboratory setting. These authors compared results

of controlled pressurization rate experiments on Ruhr sandstone and gabbro to those of controlled flow rate experiments on Ruhr sandstone. For pressurization rate of $3.0 \mathrm{MNm}^{-2} / \mathrm{s}$, the apparent tensile strength was $29.2 \mathrm{MNm}^{-2}$. For controlled flow rate of $24.3 \mathrm{MNm}^{-2}$, the apparent tensile strength was $24.3 \mathrm{MNm}^{-2}$. For further details of these very extensive 
experimental results, the original paper should be consulted.

Spence, D. A., and Turcotte, D. L. (1985) study crack propagation when the forcing fluid is magma. They found that the stress intensity factor $\gamma$ was given approximately by

$$
\gamma=k^{1 / 2}\left(A_{0}-\frac{B}{2}\right)
$$

where $A_{0}=0.5503, B=1.1006$, and $k=0.9315$. These results were also in reasonable agreement with those from earlier work by Geertsma and Haafkens (1979), where $A_{0}=0.55$, $B=1.32$, and $k=0.95$.

Boone, T. J., and Ingraffea, A. R. (1990) present numerical methods for studying hydraulically forced fracture propagation in poroelastic media. They use an equilibrium fracture model based mostly on work of Dugdale (1960) and Barenblatt (1962) to determine the fracture dimensions. The governing equations used are largely based on specialized cases from the more general work of Rice and Cleary (1976).

Sousa, J., et al. (1993) present a numerical simulation approach for hydraulic fracturing induced by Newtonian and power-law fluids. Their code is called FRANSYS (FRacture ANalysis SYStem) and it operates on a workstation with 3D graphical display. They present six viable examples of applications for their code. These include penny-shaped cracks with and without leakoff, penny-shaped crack pressure distributions, an inclined elliptical crack, perforated wellbore with Newtonian fluid, and elliptical fractures with power-law fluid.

Durham, W. B., and Bonner, B. P. (1994) treat the interactions of fluid flow and selfpropping at high effective pressure and in slightly offset joints.

Figure 4 shows some plots of data on time-dependent fracture closure due to applied stress in Westerly Granite as discussed in the work by Durham and Bonner (1994).

Renshaw, C. E., and Harvey, C. F. (1994) study fracture propagation velocity in a poroelastic medium. They emphasize the distinction between natural and induced fractures. The rate at which fluid can flow from saturated rock into void space created by fracture growth limits the propagation rate of a natural hydraulic fracture. They show that the governing equations for such natural fractures are controlled mostly by three factors: (a) the hydraulic conductivity, (b) the storage coefficient, and (c) the initial flaw length.

Falk, M. A., et al. (2001) treat dynamic fracture simulations involving cohesive surfaces. They conclude that simulations of crack branching with cohesive surfaces between elements are often not well-posed in the sense that results continually change with needed mesh 


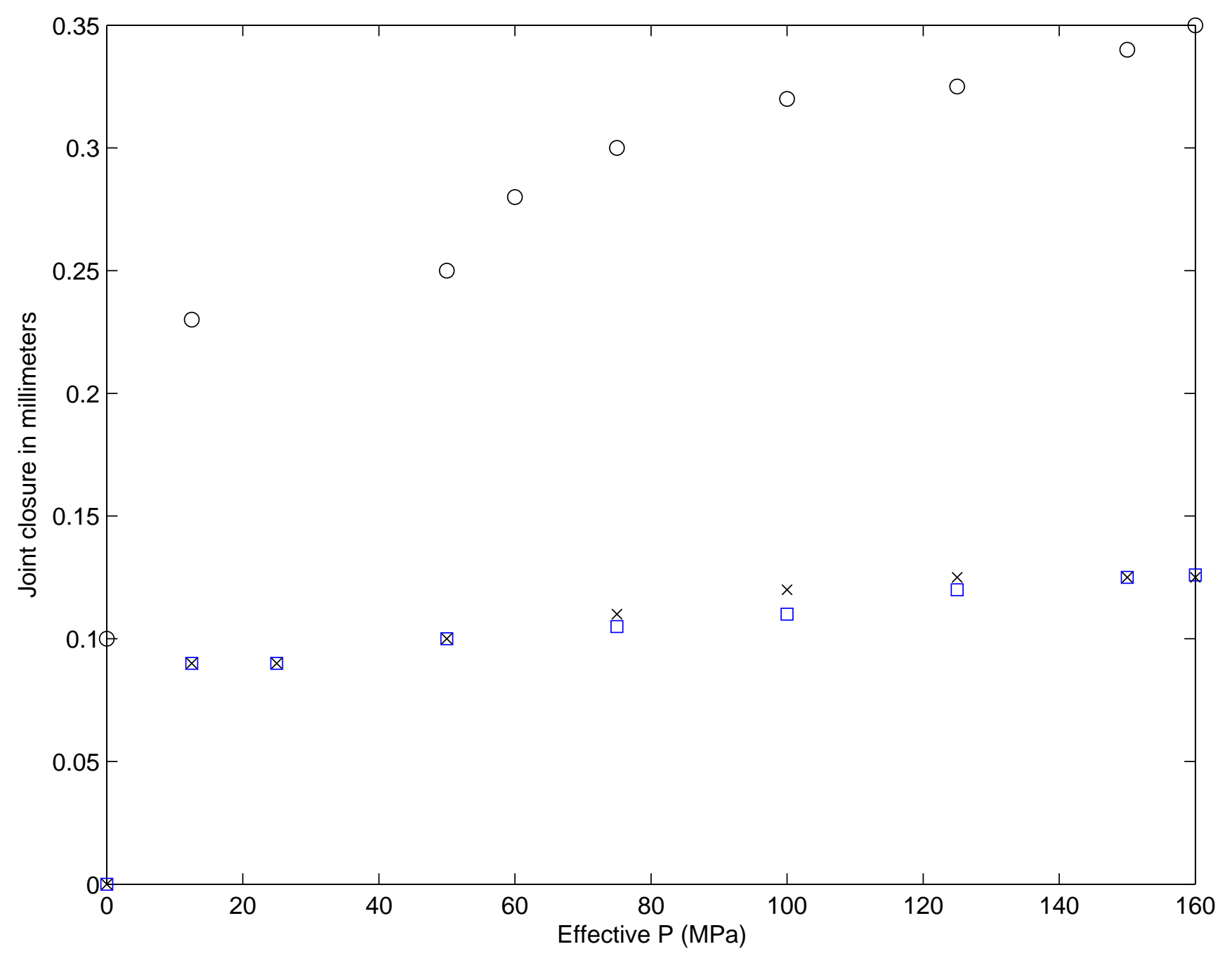

FIG. 4: Plot of crack closure versus applied stress using data from Durham and Bonner (1994) for Westerly Granite. Open circles are for Sample 1 offset, black x's are for Sample 1 mated, and blue squares are for Sample 2 mated.

refinements.

Shet, C., and Chandra, N. (2002), treat the energy balance of cohesive zone models when being used to simulate creation of new fractures. They show that the plastic energy in the material surrounding a crack is not accounted for correctly in the cohesive energy balance. So cohesive zone energy includes all the inelastic energy - such as energy required for cavitation, grain bridging, internal sliding, and surface energy, but excludes all inelastic strain energy in the bounding material.

Detournay, E. (2004) discusses propagation characteristics of fluid-driven fractures in 
otherwise impermeable rocks. The analysis focuses on the important distinction that the energy expended to create new fracture surfaces in rock is small compared to the energy dissipated in viscous flow, whereas viscous dissipation is small in comparison to the energy dissipated at the crack tip. The work also discusses significant differences between two- and three-dimensional cracks.

Bunger, A. P, and Detournay, E. (2008), provide experimental results validating the predicted tip asymptotics for a fluid-driven crack. This work brings together theory, computations, and experimental results, and shows graphical comparisons of expermential results to boundary layer solutions. Results show that the tip region of a fluid-driven crack is dominated by the viscous dissipation asymptote rather than by the classical lnear elastic fracture mechanics asymptote, as had been previously predicted by this groups' earlier work.

Peirce, A., and Detournay, E. (2008a) apply level set methods to studies of hydraulically driven fractures, while Peirce, A., and Detournay, E. (2008b) apply an Eulerian moving front algorithm for modeling hydraulically driven fractures.

Tagirova, V. R. (2009) treats hydraulic fractures when the injected fluid is non-Newtonian via an exact self-similar solution constructed when the fluid flow rate is known at the fracture inlet. Results show that use of a non-Newtonian hydraulic fracturing fluid may increase the resulting hydraulic fracture's propagation speed. For simple shear flows a complete solution of the problem can be obtained.

Carrier, B., and Granet, S. (2012) use the cohesive zone model to construct a numerical method for various hydraulic fracture problems in already permeable media. Four propagation regimes are considered, including: toughness-fracture storage, toughness-leak-off, viscosity fracture storage, and cases dominateed by viscosity-leak-off.

Saevik, P. N., et al. (2013) consider permeability upscaling methods in fractured media. Main results presented are for the differential scheme, and modified versions of both the symmetric and asymmetric self-consistent schemes. Advantages of the methods developed include avoidance of singularities found in some earlier versions, and also fewer parameters are needed to evaluate their formulas. 


\section{Summary and Conclusions}

Nonlinear pressure effects are observed in loosely consolidated sands, as was observed early on by Athy (1930). Other nonlinear effects occur in highly fractured materials, both near the surface of the earth and at greater depths. Recent work by Einav (2007a,b) provides a rather different point of view on such behavior, while the earlier work of Zimmerman, Somerton, and King (1986), Sayers and Kachanov (1995), Schoenberg and Sayers (1995), and Schoenberg (2002) - among many others discussed here - have all contributed very useful insights into the nonlinear behavior of many of these systems. Work on these systems will no doubt continue as more and different types of examples of such systems appear in data sets that need to be analyzed and understood for various practical reasons.

\section{REFERENCES}

Athy, L. F. (1930), "Density, porosity, \& compaction of sedimentary rocks," Am. Assoc. Petroleum Geologists 14, 1-24. Citations: 1220.

Bandis, S. C., Lumsden, A. C., and Barton, N. R. (1983), "Fundamentals of rock joint deformation," Int. J. Rock. Mech. Min. Sci. \& Geomech. Abstr. 20, 249-268. Citations: 1065

Barton, N., Bandis, S., and Bakhtar, K. (1985), "Strength, deformation, and conductivity coupling of rock joints," Int. J. Rock Mech. Min. Sci. $\&$ Geomech. Abst. 22, 121-140. Citations: 918.

Barenblatt, G. I. (1962), "Mathematical theory of equilibrium cracks in brittle fracture," Adv. Appl. Mech. 7, Academic Press, 55-129. Citations: 3521.

Berryman, J. G. (1992), "Single-scattering approximations for coefficients in Biot's equations of poroelasticity," Acoust. Soc. Am. 91, 551-571. Citations: 196.

Berryman, J. G., Pride, S. R., and Wang, H. F. (2002), "A differential scheme for elastic properties of rocks with dry or saturated cracks," Geophys. J. Int. 151, 597-611. Citations: 72.

Berryman, J. G., and Grechka, V. (2006), "Random polycrystals of grains containing cracks: Model of quasistatic elastic behavior for fractured systems," J. Appl. Phys. 100, 113527. Berryman, J. G., and Nakagawa, S. (2010), "Inverse problem in anisotropic poroelasticity: Drained constants from undrained ultrasound measurements," J. Acoust. Soc. Am. 127, $720-729$. 
Berryman, J. G. (2012), "Poroelastic response of orthotropic fractured porous media," Transp. Porous Media 93, 293-307.

Berryman, J. G. (2012), "Evaluating bounds and estimators for constants of random polycrystals composed of orthotropic elastic materials," Int. J. Engng. Sci. 58, 11-20.

Boone, T. J., and Ingraffea, A. R. (1990), "A numerical procedure for simulation of hydraulically driven facture propagation in poroelastic media," Int. J. Num. Analytical Methods in Geomechanics 14, 27-47. Citations: 127.

Brace, W. F. (1960), "An extension of the Griffith theory of fracture to rocks," J. Geophys. Res. 65, 3477-3480. Citations: 156.

Bristow, J. R. (1960), "Microcracks, and the static and dynamic elastic constants of annealed and heavily cold-worked metals," British J. Appl. Phys. 11, 81-85. Citations: 255.

Broberg, K. B. (1971), "Crack-growth criteria and non-linear fracture mechanics," J. Mech. Phys. Soids 19, 407-418. Citations: 211.

Brown, E. T., Bray, J. W., and Santarelli, J. T. (1989), "Influence of stress-dependent elastic moduli on stresses and strains around axisymmetric boreholes," Rock Mech. and Rock Engineering 22, 189-203. Citations: 48.

Budiansky, B., and O'Connell, R. J. (1976), "Elastic moduli of a cracked solid," Int. J. Solids Structures 12, 81-97. Citations: 1704.

Bunger, A. P., and Detournay, E. (2008), "Experimental validation of the tip asymptotics for a fluid-driven crack," J. Mech. Phys. Solids 56, 3101-3115. Citations: 21.

Bunger, A. P., Gordeliy, E., and Detournay, E. (2013), "Comparison between laboratory experiments and coupled simulations of saucer-shaped hydraulic fractures in homogeneous brittle-elastic solids," J. Mech. Phys. Solids 61, 1636-1654.

Carrier, B., and Granet, S. (2012), "Numerical modeling of the hydraulic fracture problem in permeable media using a cohesive zone model," Engineering Fracture Mech. 79, 312-328. Citations: 61.

Chen, C. Y., and Horne, R. N. (2006), "Two-phase flow in rough-walled fractures: Experiments and a flow structure model," Water Resources Res. 42, W03430. Citations: 30.

Cheng, A. H.-D., and Liggett, J. A. (1984a), "Boundary integral equation method for linear porous-elasticity with applications to soil consolidation," Int. J. Num. Methods Engng. 20, 255-278. Citations: 107. 
Cheng, A. H.-D., and Liggett, J. A. (1984b), "Boundary integral equation method for linear porous-elasticity with applications to fracture propagation," Int. J. Num. Methods Engng. 20, 279-296. Citations: 37.

Daley, T. M., Schoenberg, M. A., Rutqvist, J., and Nihei, K. T. (2006), "Fractured reservoirs: An analysis of coupled elastodynamic and permeability changes from pore-pressure variation," Geophysics 71, O33-O41. Citations: 30.

Das, A., Nguyen, G. D., and Einav, I. (2011), "Compaction bands due to grain crushing in porous rocks: A theoretical approach based on breakage mechanics," J. Geophys. Res. 116, B08203. Citations: 30.

David, E. C., and Zimmerman, R. W. (2012), "Porestructure model for elastic wave velocities in fluid-satureated sandstones," J. Geophys. Res. 117, B07210.

Detournay, E. (2004), "Propagation regimes of fluid-driven fractures in impermeable rocks," Int. J. Geomech. 4, 35-45. Citations: 203.

Dugdale, D. S.(1960), "Yielding of steel sheets constaining slits," J. Mech. Phys. Solids 8, 100-104. Citations: 5541.

Durham, W. B., and Bonner, B. P. (1994), "Self-propping and fluid flow in slightly offset joints at high effective pressures," J. Geophys. Res. 99 (B5), 9391-9399. Citations: 93.

Eberhart-Phillips, D., Han,. D.-H., and Zoback, M. D. (1989), "Empirical relationships among seismic velocity, effective pressure, porosity, and clay content in sandstones," Geophysics 54, 82-89. Citations: 348.

Einav, I. (2007a), "Breakage mechanics - Part I: Theory," J. Mech. Phys. Solids 55, 12741297. Citations: 174 .

Einav, I. (2007b), "Breakage mechanics - Part II: Modeling granular materials," J. Mech. Phys. Solids 55, 1298-1320. Citations: 82.

Elliott, H. A. (1947), "An analysis of the conditions for rupture due to Griffith cracks," Proc. Phys. Soc. 59, 208-223. Citations: 98.

Falk, M. A., Needleman, A., and Rice, J. R. (2001), "A critical evaluation of dynamic fracture simulations using cohesive surfaces," J. de Phys. IV 11, 43-52. Citations: 49.

Geertsma, J., and Haafkens, R. (1979), "A comparison of the theories for predicting width and extent of vertical hydraulically induced fractures," J. Energy Resour. Technol. 101, 8-19. Citations: 115. 
Grechka, V., and Kachanov, M. (2006a), "Effective elasticity of rocks with closely spaced and intersecting cracks," Geophysics 71, D85-D91.

Grechka, V., and Kachanov, M. (2006b), "Seismic characterization of multiple fracture sets: Does orthotropy suffice?" Geophysics 71, D93-D105. Citations: 55.

Grechka, V., Vasconcelos, I., and Kachanov, M. (2006), "Influence of crack shape on effective elasticity of fractured rocks," Geophysics 71, D153-D160. Citations: 39.

Griffith, A. A. (1924), "The phenomena of rupture and flow in solids," Phil. Trans. Roy. Soc. A 221, 163-197. Citations: 4722.

Han, D., Nur, A., and Morgan, D. (1986), "Effects of porosity and clay content on wave velocities in sandstones," Geophysics 51, 2093-2107. Citations: 928.

Hashin, Z., and Shtrikman, S. (1961), "Note on a variational approach to the theory of composite elastic materials," J. Franklin Inst. 271, 336-341. Citations: 145.

Howard, G. C., and Fast, C. R. (1957), "Optimum fluid characteristics for fracture extension," Drilling and Production Practice 24, 261-270. Citations: 228.

Johnson, L. R. (2014), "A source model for induced earthquakes at the Geysers Geothermal Reservoir," Pure Appl. Geophys. 171, 1625-1640.

Johnson. P. A., and Rasolofosaon, P. N. J. (1996), "Manifestation of nonlinear elasticity in rock: Convincing evidence over large frequency and strain intervals from laboratory studies," Nonlinear Processes in Geophysics 3, 77-88. Citations: 80.

Kachanov, M. (1980), "Continuum model of medium with cracks," J. Engng. Mech. ASCE 106, 1039-1051. Citations: 378.

Katsuki, D., Gutierrez, M., and Almrabat, A. (2014), "Stress-dependent elastic wave velocity of microfractured sandstone," Int. J. Num. Anal. Meth. Geomech. 38, 441-456.

Lian, P. Q., Cheng, L. S., and Liu, L. F. (2011), "Characteristics of productivity curves in abnormal high-pressure reservoirs," Petroleum Science and Technology 29, 109-120.

Liu, H. H., Rutqvist, J., and Berryman, J. G. (2009), "On the relationship between stress and strain for porous and fractured rock," Int. J. Rock. Mech. ES Mining Sci. 46, 289-296. Citations: 38.

Liu, H. H., and Rutqvist, J. (2013), "Coupled hydro-mechanical processes associated with multiphase flow in a dual-continuum system: Formulations and an application," Rock Mech. Rock Eng. 46, 1103-1112. 
Liu, H. H., Wei, M.-Y., and Rutqvist, J. (2013), "Normal-stress dependence of fracture hydraulic properties including two-phase flow properties," Hydrogeology J. 21, 371-382.

Malama, B., and Kulatilake, P. H. S. M. (2003), "Models for normal fracture deformation under compressive loading," Int. J. Rock Mech. \& Mining Sci 40, 893-901. Citations: 28. Matsuki, K., Wang, E. Q., Sakaguchi, K., and Okumura, K. (2001), "Time-dependent closure of a fracture with rough surfaces under constant normal stress," Int. J. Rock Mech. Min. Sci. 38, 607-619.

Mavko, G., Mukerji, T., and Dvorkin, J. (1998), The Rock Physics Handbook, Cambridge Univ. Press (1998), p. 28. Citations: 2721.

Neuzil, C. E. (2003), "Hydromechanical coupling in geologic processes," Hydrogeology Journal 11, 41-83. Citations: 115.

Nilsson, F. (1973), "A path-independent integral for transient crack problems," Int. J. Solids Struct. 9, 1107-1115. Citations: 51.

Orowan E. (1949), "Fracture and strength of solids," Reports on Progress in Physics 12, 185-232. Citations: 1147.

Peirce A., and Detournay, E. (2008a), "An implicit level set method for modeling hydraulically driven fractures," Computer Methods in Applied Mechanics and Engineering 197, 28582885.

Peirce A., and Detournay, E. (2008b), “An Eulerian moving front algorithm with weak-form tip asymptotics for modeling hydraulically driven fractures," Commun. Num. Methods Engng. 25, 185-200.

Renshaw, C. E., and Harvey, C. F. (1994), "Propagation velocity of a natural hydraulic fracture in a poroelastic medium," J. Geophys. Res. 99, 21667-21677.

Reuss, A. (1929), "Berechung der Fliessgrenze von Mischkristallen," Z. Angew. Mat. Mech. 9, 55. Citations: 2727.

Rice, J. R. (1999), “A path independent integral and approximate analysis of strain concentration by notches and cracks," J. Appl. Mech. 35, 379-386. Citations: 6236.

Rice, J. R., and Cleary, M. P. (1976), "Some basic stress diffusion solutions for fluid-saturated porous media with compressible constitutents," Rev. Geophys. 14, 227-241. Citations: 1395.

Rubin, M. B. (1991), "Simple, convenient isotropic failure surface," J. Engng. Mech. 117, 348-369. Citations: 36. 
Rubin, M. B., and Einav, I. (2011), "A large deformation breakage model of granular materials including porosity and inelastic distortional deformation rate," Int. J. Engng. Sci. 49, 1151-1169.

Sack, R. A. (1946), "Extension of Griffith's theory of rupture to three dimensions," Proc. Phys. Soc. 58, 729-736. Citations: 316.

Saevik, P. N., Berre, I., Jakobsen, M., and Lien, M. (2013), "A 3D computational study of effective medium methods applied to fracture media," Transport in Porous Media 100, $115-142$.

Salimi, H., and Bruining, H. (2010), "Upscaling in vertically fractured oil reservoirs using homogenization," Transport in Porous Media 84, 21-53.

Sayers, C. M., and den Boer, L. D. (2012), "Characterizaing production-induced anisotropy of fractured reservoirs having multiple fracture sets," Geophys. Prospecting 60, 919-939. Sayers, C. M., and Kachanov, M. (1991), "A simple technique for finding effective elastic constants of cracked solids for arbitrary crack orientation statistics," Int. J. Solids Structures 27, 671-680. Citations: 581.

Sayers, C. M., and Kachanov, M. (1995), "Microcrack-induced elastic-wave anisotropy of brittle rocks," J. Geophys. Res. 100, 4149-4156. Citations: 308.

Schoenberg, M., and Sayers, C. M. (1995), "Seismic anisotropy of fractured rock," Geophysics 60, 204-211. Citations: 581.

Schoenberg, M. (2002), "Time-dependent anisotropy induced by pore pressure variation in fractured rock," J. Seismic Exploration 11, 83-105.

Sevostianov, I., and Kachanov, M. (2012), "Is the concept of average shape legitimate, for a mixture of inclusioins of diverse shapes?" Int. J. Solids Structures 49, 3242-3254.

Sevostianov, I., and Kachanov, M. (2013), "Non-interaction approximation in the problem of effective properties," in Effective Properties of Heterogeneous Materials, Solid Mechanics and Its Applications 193, pp. 1-95.

Shapiro. S. A. (2003), "Elastic piezosensitivity of porous and fractured rocks," Geophysics 68, 482-486. Citations: 63.

Shet, C., and Chandra, N. (2002), "Analysis of energy balance when using cohesive zone models to simulate fracture processes," J. Engineering Materials \& Technology - Trans. ASME 124, 440-450. Citations: 71. 
Sousa, J., Carter, B., and Ingraffea, A., (1993), "Numerical simulatio of 3D hydraulic fracture using Newtonian and power-law fluids," Int. J. of Rock Mech. and Mining Sciences 30, 1265-1271. Citations: 34.

Spence, D. A., and Turcotte, D. L. (1985), "Magma driven propagation of cracks," J. Geophys. Res. 90 (B1), 575-580. Citations: 244.

Tagirova, V. R. (2009), "Hydraulic fracture crack propagation driven by a non-Newtonian fluid," Moscow Univ. Mech. Bulletin 64, 135-142.

Tsvankin, I. (1997), "Anisotropic parameters and P-wave velocity for orthorhombic media," Geophysics 62, 1292-1309. Citations: 332.

Tsvankin, I., Gaiser, J., Grechka, V., van der Baan, M., and Thomsen, L. (2010), "Seismic anisotropy in exploration and reservoir characterization: An overview", Geophys. 75, A15A29. Citations: 84.

Tullis, J., and Yund, R. A. (1980), "Hydrolytic weakening of experimentally deformed Westerly granite and Hale albite rock," J. Struct. Geol. 2, 439-451. Citations: 184.

Tullis, J., and Yund, R. A. (1989), "Hydrolytic weakening of quartz aggregates: The effects of water and pressure on recovery," Geophys. Res. Lett. 16, 1343-1346. Citations: 53.

Tuncay, K., and Corapcioglu, M. Y. (1996a), "Wave propagation in fractured porous media," Transport in Porous Media 23, 237-258. Citations: 33.

Tuncay, K., and Corapcioglu, M. Y. (1996b), "Body waves in fractured porous media saturated by two immiscible Newtonian fluids," Transport in Porous Media 23, 259-273. Citations: 24 .

van der Kamp, G., and Gale, J. E. (1983), "Theory of earth tide and barometric effects in porous formations with compressible grains," Water Resources Research 19, 538-544. Citations: 194.

Vasco, D. W., Dutta, A., Gupta, R., Behrens, R., and Condon, P. (2004), "Seismic imaging of reservoir flow properties: Time-lapse pressure changes," Geophysics 69, 511-521. Citations: 44.

Vasco, D. W., and Karasaki, K. (2001), "Inversion of pressure observations: An integral formulation," J. Hydrology 253, 27-40. Citations: 29.

Voigt, W., (1928), Lehrbuch der Kristallphysik Teubner, Leipzig, p, 962.

Wang, H. F. (1997), "Effects of deviatoric stress on undrained pore pressure response to fault slip," J. Geophys. Res. 102, 17943-17950. Citations: 35. 
Warren, J. E., and Root, P. J. (1963), "The behavior of naturally fractured reservoirs," Soc. Pet. Eng. J. 228, 245-255. Citations: 2419.

White, J. W. (1973), "An invariant description of failure for an isotropic medium," $J$. Geophys. Res. 78, 2438-2441.

Wyble, D. O. (1958), "Effect of applied pressure on the conductivity, porosity, and permeability of sandstones," J. Petrol. Tech. 10, 57-59. (Also, SPE 1081-G, Technical Note 2022 and PhD thesis, Penn State Univ.) Citations: 103.

Zhang, Y., Sayers, C. M., and Adachi, J. I. (2009), "The use of effective medium theories for seismic wave propagation and fluid flow in fractured reservoirs under pressure," Geophys. J. Int. 177, 205-221.

Zhou, X., and Burbey, T. J. (2014), "Fluid effect on hydraulic fracture propgation behavior: A Comparison between water and supercritical $\mathrm{CO}_{2}$-like fluid," Geofluids 14, 174-188.

Zimmerman, R. W. (1991), "Compressibility of Sandstones," Elsevier, p. 130.

Zimmerman, R. W., Chen, G., and Bodvarsson, G. S. (1992), "A dual-porosity reservoir model with an improved coupling term," Proceeding, 17th Workshop on Geothermal Reservoir Engineering, Stanford University (1992).

Zimmerman, R. W., Somerton, W., and King, M. S. (1986), "Compressibility of porous rocks," J. Geophys. Res. 91, 12765-12777. Citations: 233.

Zoback, M. D., Rummel, F., Jung, R., Raleigh, C. B. (1977), "Laboratory hydraulic fracturing experiments in intact and pre-fractured rock," Int. J. Rock Mech. Mining Sci. and Geomechanics Abstracts 14, 49-58. Citations: 114. 